\title{
STABILITY OF SOLUTIONS OF SOME CLASSES OF NONLINEAR OPERATOR EQUATIONS
}

\author{
R. H. KASRIEL AND M. Z. NASHED
}

The following questions are of interest in many applications of nonlinear operator equations: Under what conditions will the existence of a solution be preserved with a slight change of the operator? How much of a change results in the solution?

These questions have been studied in many classical settings related to algebraic equations, differential and integral equations, etc. In the last two decades, some of these investigations have been unified in the context of functional analysis. Related questions have been studied by Lonseth [8] for linear problems and by Block [1], Krasnosel'skii [6] and others for nonlinear equations. These questions also rise in connection with computational processes. For example, see Mihlin [9] and Urabe [12] for linear and nonlinear equations respectively.

It is obvious that these questions are of a very general nature and that their investigation depends on the class of operators considered and on the notion of a "slight change." The purpose of this paper is to formulate and investigate these questions in terms of specific topologies on certain classes of nonlinear operators. Some results on the lightness and openness of certain mappings are obtained as a byproduct of our investigation.

1. Preliminaries. Let $B$ be a Banach space and let $K$ be a class of continuous mappings from open subsets of $B$ into $B$. By $S\left(x_{0}, r\right)$, we shall mean the open sphere with radius $r$ and center $x_{0}$. Let $\mathfrak{I}$ be a topology for $K$. Let $A_{0} \in K$ and $u_{0} \in B$. We say that a solution $x_{0}$ of the equation

$$
A_{0} x=u_{0}
$$

is stable with respect to $(K, \mathfrak{T})$ provided that for each $r>0$, there exists a neighborhood $\Omega$ of $A_{0}$ (rel. to $(K, \mathfrak{I})$ ) and $d>0$ such that for each $A \in \Omega$ and $u \in S\left(u_{0}, d\right)$, the equation

$$
A x=u
$$

has a solution in $S\left(x_{0}, r\right)$. If for each $x_{0}$ in the domain of $A_{0}, x_{0}$ is a

Received by the editors August 9, 1965 and, in revised form, January 28, 1966. 
stable solution of the equation $A_{0} x=A_{0} x_{0}$, we then say that $A_{0}$ is a stable operator with respect to $(K, \mathfrak{I})$.

It should be remarked that even if a stable solution of (1) happens to be unique, there is no guarantee that the disturbed equation will have a unique solution. However, suppose because of the nature of the particular equation and the class of mappings studied, each of the disturbed equations has a unique solution. Then stability implies a form of continuous dependence of the solution on the operator and the right member.

We now define two classes of mappings from open subsets of $B$ into $B$. By $K^{\prime}$, we shall mean the class of all such mappings that are continuous. By $K^{\prime \prime}$, we shall mean the collection of all $A \in K^{\prime}$ such that $I-A$ is completely continuous (where $I$ is the identity mapping). A convenient way to generate topologies on $K^{\prime}$ and $K^{\prime \prime}$, appropriate for our purposes, is to make use of $\Omega$-neighborhoods as introduced by Ehrmann in [2]. The $\Omega_{L}$-neighborhoods for $K^{\prime}$, to be defined in $\S 2$ in terms of a Lipschitz condition, are as discussed in \$2 of [2] except for slight notational modification. The $\Omega_{U}$-neighborhoods for $K^{\prime \prime}$, defined in $\$ 3$, generate a topology related to uniform convergence on bounded subsets. Note that questions of stability for $K^{\prime}$ and $K^{\prime \prime}$ can be reduced to considerations of equations of the form

$$
A_{0} x=\theta \text {. }
$$

2. The space $\left(K^{\prime}, \mathfrak{l}\right)$. We shall call $\left(x_{0}, A_{0}, r\right)$ an admissible triple provided that $A_{0} \in K^{\prime}$ and the closed sphere $\bar{S}\left(x_{0}, r\right) \subset D$, the domain of $A_{0}$. By $\Omega_{L}\left(x_{0}, A_{0}, r, a, b\right)$, for a given admissible triple $\left(x_{0}, A_{0}, r\right)$, $a>0$, and $b>0$, we mean the collection of all mappings $A$ in $K^{\prime}$ for which

$$
\begin{gathered}
\left(x_{0}, A, r\right) \text { is an admissible triple, } \\
\left\|\left(A-A_{0}\right) x_{0}\right\| \leqq a \\
\left\|\left(A-A_{0}\right) x-\left(A-A_{0}\right) x_{0}\right\| \leqq b\left\|x-x_{0}\right\| \text { for } x \in \bar{S}\left(x_{0}, r\right) .
\end{gathered}
$$

We then define $\mathfrak{R}$ to be the topology generated by taking the collection of all $\Omega_{L}$-neighborhoods as a subbase.

Recall that the Lipschitzian norm of an operator $A$ on a set $S$ is defined by

$$
\|A\|_{S}=\sup \left\{\frac{\|A x-A y\|}{\|x-y\|}: x \neq y ; x, y \in S\right\} .
$$

The subscript $S$ will be omitted when there is no chance of confusion. If $\|A\|$ is finite then $A$ is said to be Lipschitzian on $S$. 
Using a standard argument involving a local form of Banach's contractive principle, the following theorem is easy to prove.

2.1. A stability theorem for the class $\left(K^{\prime}, \Omega\right)$. Let $A_{0} \in K^{\prime}$. Suppose on the closed sphere $\bar{S}\left(x_{0}, r\right)$,

$$
\begin{aligned}
\left\|I-A_{0}\right\| & =k<1, \\
\left\|A_{0} x_{0}\right\| & \leqq r(1-k) .
\end{aligned}
$$

Then, the unique solution in $\bar{S}\left(x_{0}, r\right)$ of the equation $A_{0} x=\theta$ is stable with respect to $\left(K^{\prime}, \Omega\right)$.

Next, we consider certain classes of operator equations whose stability can be established with the aid of this theorem.

2.2. Operators with bounded Fréchet derivatives. If an operator $A$ has a bounded Fréchet derivative $A_{x}^{\prime}$ on a convex domain $D$, then $\|A\|_{D}$ $=\sup \left\{\left\|A_{x}^{\prime}\right\|: x \in D\right\}$. (See e.g., pp. 660-661 in [4]). In this case, we may replace condition (6) in the previous theorem by the condition $\sup \left\{\left\|I-A_{x}^{\prime}\right\|: x \in D\right\}=k<1$.

2.3. Stability of strongly monotone operator equations. Let $A_{0}$ be a mapping from an open subset $D$ of a Hilbert space $H$ in to $H$. Suppose $A_{0}$ is Lipschitzian on $\bar{S}\left(x_{0}, r\right) \subset D$ with norm $M$ and $A_{0}$ is strongly monotonic on $\bar{S}\left(x_{0}, r\right)$; i.e., there exists an $m>0$ such that for all $x, y$ in $\bar{S}\left(x_{0}, r\right)$,

$$
\operatorname{Re}\left\langle A_{0} x-A_{0} y, x-y\right\rangle \geqq m\|x-y\|^{2} .
$$

If, in addition, we assume that for some $\lambda$ in the open interval $(0$, $\left.2 m / M^{2}\right)$,

$$
\lambda\left\|A_{0} x_{0}\right\| \leqq r\left(1-\left\|I-\lambda A_{0}\right\|\right),
$$

then the unique solution in $\bar{S}\left(x_{0}, r\right)$ of equation (1)' is stable with respect to $\left(K^{\prime}, \mathfrak{l}\right)$.

Proof. For any $x$ and $y$ in $\bar{S}\left(x_{0}, r_{0}\right)$ and $\lambda>0$,

$$
\begin{aligned}
& \left\|\left(x-\lambda A_{0} x\right)-\left(y-\lambda A_{0} y\right)\right\|^{2} \\
& \quad=\|x-y\|^{2}+\lambda^{2}\left\|A_{0} x-A_{0} y\right\|^{2}-2 \lambda \operatorname{Re}\left\langle A_{0} x-A_{0} y, x-y\right\rangle \\
& \leqq\left(1+\lambda^{2} M^{2}-2 \lambda m\right)\|x-y\|^{2} .
\end{aligned}
$$

Now note that if $\lambda \in\left(0,2 m / M^{2}\right)$, then $1+\lambda^{2} M^{2}-2 \lambda m<1$. Hence $\left\|I-\lambda A_{0}\right\|<1$ on $\bar{S}\left(x_{0}, r_{0}\right)$. Since we are assuming that (9) holds, then it follows from 2.1 that the equation $\lambda A_{0} x=\theta$ has a unique solution in $\bar{S}\left(x_{0}, r_{0}\right)$ which is stable. Hence so does $A_{0} x=\theta$.

REMARK. If $A_{0}$ has a Gâteaux differential on a convex set $S$, then using a mean value theorem argument, it follows that for any $x, y \in S$, $h=y-x$, 


$$
\operatorname{Re}\left\langle A_{0} y-A_{0} x, y-x\right\rangle=\left.\frac{d}{d s}\left\langle A_{0}(x+\tau h+s h), h\right\rangle\right|_{s=0}
$$

for some $0<\tau<1$. (See Lemma 3.2 in Vaŭnberg [13] or the proof of Theorem 6 in Minty [10].) Hence a sufficient condition for (8) to hold on a convex set $S$ is that there exists $m>0$ such that for all $z$, $z+h \in S$,

$$
\left.\frac{d}{d t} \operatorname{Re}\left\langle A_{0}(z+t h), h\right\rangle\right|_{t=0} \geqq m\|h\|^{2} .
$$

3. The space $\left(K^{\prime \prime}, \mathfrak{u}\right)$. Let $A_{0} \in K^{\prime \prime}($ See $\S 1)$ and let $\left(x_{0}, A_{0}, r\right)$ be an admissible triple (See $\S 2$.) By $\Omega_{U}\left(x_{0}, A_{0}, r, b\right)$ we shall mean the collection of all $A \in K^{\prime \prime}$ such that

$$
\begin{gathered}
\left(x_{0}, A, r\right) \text { is an admissible triple, } \\
\left\|\left(A-A_{0}\right) x\right\| \leqq b \text { for all } x \in \bar{S}\left(x_{0}, r\right) .
\end{gathered}
$$

$\mathfrak{U}$ is the topology on $K^{\prime \prime}$ generated by taking the collection of all such sets as a subbase.

It is of interest to note that $K^{\prime \prime}$ is closed under uniform limits on bounded subsets. (See p. 15 of [7].) Furthermore if $A_{i}, i=1,2, \cdots$ and $A_{0}$ are elements of $K^{\prime \prime}$ defined on $B$, then $\lim A_{i}=A_{0}$ (with respect to $\left.\left(K^{\prime \prime}, \mathfrak{u}\right)\right)$ if and only if the sequence of mappings $A_{i}$ converges to $A_{0}$ uniformly on bounded subsets of $B$.

3.1. The functions $R$ and $\alpha$. We define two numerical quantities that are useful in the analysis and statement of results concerning stability with respect to $\left(K^{\prime \prime}, \mathfrak{U}\right)$.

$$
R\left(x_{0}, A_{0}, r\right)=r^{-1} \sup \left\{\left\|\left(x-A_{0} x\right)-\left(x_{0}-A_{0} x_{0}\right)\right\|: x \in \bar{S}\left(x_{0}, r\right)\right\} .
$$

If $R\left(x_{0}, A_{0}, r\right)<1$ for some $r>0$, then we define

$$
\alpha\left(x_{0}, A_{0}\right)=\inf \left\{r: R\left(x_{0}, A_{0}, r\right)<1\right\} .
$$

The following observations concerning these functions will be useful later.

3.2(a). If for some $x$ for which $\left\|x-x_{0}\right\|=r>0$, it is true that $A_{0} x$ $=A_{0} x_{0}$, then $R\left(x_{0}, A_{0}, r\right) \geq 1$.

The next remark is an immediate consequence of the contrapositive of (a).

3.2(b). Let $r^{\prime}>0$. If $R\left(x_{0}, A_{0}, r\right)<1$ for each $r, 0<r<r^{\prime}$, then $A_{0} x \neq A_{0} x_{0}$ for $0<\left\|x-x_{0}\right\|<r^{\prime}$.

3.3. A stability theorem for $\left(K^{\prime \prime}, \mathfrak{U}\right)$. Let $A_{0}: D \rightarrow B$ be a mapping in $K^{\prime \prime}$. Suppose $x_{0} \in D$ is a solution of $(1)^{\prime}$ and $\alpha\left(x_{0}, A_{0}\right)=0$. Then $x_{0}$ is a stable solution of $(1)^{\prime}$ with resect to $\left(K^{\prime \prime}, \mathfrak{u}\right)$. 
We prove the theorem by proving first the stronger statement in 3.4. The proof of 3.3 then will follow from 3.4 by taking $k=0$.

3.4. Suppose $x_{0}$ is a solution of $A_{0} x=\theta$ and $\alpha\left(x_{0}, A_{0}\right)=k$. Then, for each $e>0$, there exist positive numbers $b, d$, and $r, 0<r<k+e$, with the property that if $A \in \Omega_{U}\left(x_{0}, A_{0}, r, b\right)$ and $\|u\|<d$, then the equation $A x=u$ has a solution in $\bar{S}\left(x_{0}, r\right)$.

Proof. Since $\alpha\left(x_{0}, A_{0}\right)=k$, for $e>0$, there is an $r>0$ such that $0<r<k+e$ and $R=R\left(x_{0}, A_{0}, r\right)<1$. Now choose positive numbers $b$ and $d$ such that

$$
b+d<(1-R) r .
$$

Let $A \in \Omega_{U}\left(x_{0}, A_{0}, r, b\right)$ and $u \in B$ with $\|u\|<d$. Consider the mapping $F$ on $\bar{S}\left(x_{0}, r\right)$ defined by $F x=x-A x+u$. Since $A \in K^{\prime \prime}, I-A$ and hence $F$ is completely continuous on $\bar{S}\left(x_{0}, r\right)$. Thus, if we can show that

$$
F\left(\bar{S}\left(x_{0}, r\right)\right) \subset \bar{S}\left(x_{0}, r\right),
$$

it will follow from Schauder's fixed point theorem that $F$ has a fixed point $y$ in $\bar{S}\left(x_{0}, r\right)$. But then $A y=u$. We complete the proof by verifying (15). Toward that end, let $x \in \bar{S}\left(x_{0}, r\right)$. Then, using (14), the definition of $\alpha$ and $R$,

$$
\begin{aligned}
\left\|F x-x_{0}\right\| & \leqq\left\|x-A_{0} x-x_{0}\right\|+\left\|A x-A_{0} x\right\|+\|u\| \\
& \leqq R r+b+d<R r+(1-R) r=r .
\end{aligned}
$$

3.5. REMARK. In [11], Reichbach proved, using the same fixed point principle used in 3.4, that if $A_{0} \in K^{\prime \prime}$ and $R\left(x_{0}, A_{0}, r\right)<1$, then $A_{0} x_{0}$ is an interior point of the range of $A_{0}$. This follows from the proof of 3.4 which shows that $S\left(A_{0} x_{0}, d\right) \subset A_{0}\left(\bar{S}\left(x_{0}, r\right)\right)$ provided that $d<(1-R) r$.

As a by-product of 3.3 we next obtain a result concerning light and open mappings.

3.6. A sufficient condition for a mapping to be light and open. Recall that a mapping $A: X \rightarrow Y$ is said to be open at a point $x_{0} \in X$ provided that for any open set $U$ for which $x_{0} \in U, A x_{0}$ is an interior point (rel. $Y$ ) of $A(U)$. If $A$ is open at each $x \in X$, then it follows that $A$ is an open mapping (i.e., $A(U)$ is open if $U$ is open). Note that it follows easily from the definition in $\S 1$, that stability of a mapping with respect to $(K, \mathfrak{T})$ implies its openness.

A mapping $A$ is said to be light if for each $y$ in its range, $A^{-1} y^{\prime}$ is totally disconnected (i.e., contains no nondegenerate continuum).

If in addition each point of $A^{-1} y$ is an isolated point of $A^{-1} y$, then $A$ is said to have the scattered inverse property. 
These properties are possessed by analytic mappings on regions in the complex plane and have been the subject of much investigation. See, for example, [15].

Theorem. Let $A_{0}$ be a mapping from an open subset $D \subset B$ into a Banach space $B$. Let $I-A_{0}$ be completely continuous.

(i) If $\alpha\left(x, A_{0}\right)=0$ for each $x \in D$, then $A_{0}$ is light and open.

(ii) If for each $x \in D$, there is an $r_{x}$ such that for $0<r<r_{x}, R\left(x, A_{0}, r\right)$ $<1$, then $A_{0}$ also has the scattered inverse property.

Proof. That $A_{0}$ is open follows from Theorem 3.3 and the first paragraph of this section. We next prove that $A_{0}$ is light.

Suppose there is a nondegenerate continuum $Q$ of $A_{0}^{-1}\left(A_{0} x_{0}\right)$ that contains $x_{0}$. Let $x^{\prime} \in Q-\left\{x_{0}\right\}$ and $r^{\prime}=\left\|x_{0}-x^{\prime}\right\|$. For each $r, 0<r<r^{\prime}$, we may choose $x(r) \in Q$ for which $r=\left\|x(r)-x_{0}\right\|$; for otherwise, we could find a separation of $Q$. From 3.2(a), it now follows that $R\left(x_{0}, A, r\right) \geq 1$ for $0<r<r^{\prime}$ and $\alpha\left(x_{0}, A\right) \geq r^{\prime} \neq 0$.

Assertion (ii) follows from 3.2(b).

3.7. Stable values of a continuous mapping. Another notion related to our investigation is that of stable value of a continuous mapping as discussed for example in [3], [5], and [14]. Suppose $X$ and $Y$ are normed linear spaces and $A_{0}: X \rightarrow Y$ is continuous. A point $y_{0} \in A_{0}(X)$ is called a stable value of $A_{0}$ provided that there is a $d>0$ such that $y_{0} \in A(X)$ for any con tinuous mapping $A: X \rightarrow Y$ for which $\left\|\left(A-A_{0}\right) x\right\|$ $<d$. Now note that if $A_{0}$ is a continuous mapping from an open subset $D$ of Euclidean $n$-space $E_{n}$, then $I-A_{0}$ is completely continuous and hence $A_{0} \in K^{\prime \prime}$. If further $R\left(x_{0}, A_{0}, r\right)<1$ for some $r$, it then follows from the proof of 3.4 that $A_{0} x_{0}$ is a stable value of $A_{0}$.

The authors wish to thank the referee for his helpful suggestions.

\section{REFERENCES}

1. H. D. Block, Construction of solutions and propagation of errors in nonlinear problems, Proc. Amer. Math. Soc. 4 (1953), 715-722.

2. H. Ehrmann, On implicit function theorems and the existence of solutions of nonlinear equations, Enseignement Math. 9 (1963), 129-176.

3. W. Hurewicz and H. Wallman, Dimension theory, Princeton Univ. Press, Princeton, N. J., 1948.

4. L. V. Kantorovich and G. P. Akilov, Functional analysis in normed spaces, Pergamon, New York, 1964.

5. R. H. Kasriel, Stability of certain quasi-open mappings, Duke Math. J. 28 (1962), 595-606.

6. M. A. Krasnosel'skiY, Some problems of nonlinear analysis, Amer. Math. Soc. Transl. (2) 10 (1958), 345-409.

7. - Topological methods in the theory of nonlinear integral equations, Pergamon, New York, 1964. 
8. A. T. Lonseth, The propagation of error in linear problems, Trans. Amer. Math. Soc. 62 (1947), 193-212.

9. S. G. Mihlin, Stability of some computing processes, Dokl. Akad. Nauk. SSSR 157 (1964), 271-273=Soviet Math. Dokl. 5 (1964), 931-933.

10. G. J. Minty, Monotone (nonlinear) operators in Hilbert space, Duke Math. I. 29 (1962), 341-346.

11. M. Reichbach, Fixed points and openness, Proc. Amer. Math. Soc. 12 (1961), 734-736.

12. M. Urabe, Convergence of numerical iteration in solution of equations, J. Sci. Hiroshima Univ. Ser. A 19 (1956), 479-489.

13. M. M. Valnberg, Variational methods for the study of nonlinear operators, Holden-Day, San Francisco, Calif., 1964.

14. J. Väisälä, Minimal mappings in Euclidean spaces, Ann. Acad. Sci. Fenn. Ser. AI 366 (1965), 22 pp.

15. G. T. Whyburn, Topological analysis, Princeton Univ. Press, Princeton, N. J., 1964.

Georgia Institute of Technology 